\title{
X-ray pinhole camera setups used in the Atomki ECR Laboratory for plasma diagnostics
}

\author{
R. Rácz, ${ }^{1, a)}$ S. Biri, ${ }^{1}$ J. Pálinkás, ${ }^{1}$ D. Mascali, ${ }^{2}$ G. Castro, ${ }^{2}$ C. Caliri, ${ }^{2}$ F. P. Romano,,${ }^{2,3}$ \\ and S. Gammino ${ }^{2}$ \\ ${ }^{1}$ Institute for Nuclear Research (Atomki), Hungarian Academy of Sciences, Bem tér 18/C, H-4026 Debrecen, \\ Hungary \\ ${ }^{2}$ Instituto Nazionale di Fisica Nucleare-Laboratori Nazionali del Sud, via S. Sofia 62, 95123 Catania, Italy \\ ${ }^{3}$ CNR, Istituto per i Beni Archeologici e Monumentali, Via Biblioteca 4, 95124 Catania, Italy
}

(Presented 27 August 2015; received 21 August 2015; accepted 30 September 2015; published online 23 December 2015)

\begin{abstract}
Imaging of the electron cyclotron resonance (ECR) plasmas by using CCD camera in combination with a pinhole is a non-destructive diagnostics method to record the strongly inhomogeneous spatial density distribution of the X-ray emitted by the plasma and by the chamber walls. This method can provide information on the location of the collisions between warm electrons and multiple charged ions/atoms, opening the possibility to investigate the direct effect of the ion source tuning parameters to the plasma structure. The first successful experiment with a pinhole X-ray camera was carried out in the Atomki ECR Laboratory more than 10 years ago. The goal of that experiment was to make the first ECR X-ray photos and to carry out simple studies on the effect of some setting parameters (magnetic field, extraction, disc voltage, gas mixing, etc.). Recently, intensive efforts were taken to investigate now the effect of different RF resonant modes to the plasma structure. Comparing to the 2002 experiment, this campaign used wider instrumental stock: CCD camera with a lead pinhole was placed at the injection side allowing X-ray imaging and beam extraction simultaneously. Additionally, Silicon Drift Detector (SDD) and High Purity Germanium (HPGe) detectors were installed to characterize the volumetric X-ray emission rate caused by the warm and hot electron domains. In this paper, detailed comparison study on the two X-ray camera and detector setups and also on the technical and scientific goals of the experiments is presented. (C) 2015 AIP Publishing LLC. [http://dx.doi.org/10.1063/1.4933085]
\end{abstract}

\section{INTRODUCTION}

Electron Cyclotron Resonance Ion Sources (ECRIS) provide worldwide highly charged ion beams for high energy accelerators and also the low energy ion beam of the source can be used in various fields of science (e.g., in atomic physics research, material science). Being the parameters of the extracted ion beam strongly determined by the quality of the ECR plasma, deep understanding of the plasma and its behavior is essential. Therefore, since the development of the pioneer ECRISs, different plasma diagnostics methods were developed and adapted.

Instead of the frequently used local plasma diagnostics methods (Langmuir probes, see, e.g., Ref. 1), the global diagnostics are non-destructive and are based on the detection and evaluation of the electromagnetic wave (EMW) emission of the plasma.

The most important electron component of the plasma (for highly charged ions) is the so called warm electron portion having energy between about 3 and $10 \mathrm{keV}$. The atoms and ions are excited and ionized step-by-step by the warm electrons and produce characteristic X-rays. Imaging of the plasma by using the combination of special CCD camera and a pinhole

Note: Contributed paper, published as part of the Proceedings of the 16th International Conference on Ion Sources, New York, New York, USA, August 2015.

a)rracz@atomki.hu allows recording the strongly inhomogeneous X-ray emission corresponding to the axially projected perspective snapshot of the warm electrons. ${ }^{2} \mathrm{X}$-ray imaging by $\sim 0.1 \mathrm{~mm}$ in diameter $(w)$ pinhole is ideal: the wavelength $(\lambda)$ of the characteristic $X$ ray lower than $1 \mathrm{~nm}$ therefore easy to provide focal distance $\left(\mathrm{d}_{\mathrm{f}}\right)$ keeping $d_{f} \times \lambda \ll w$ causing negligible diffraction effects. Furthermore, the spatial resolution of the optics is reciprocally proportional with $w$ and it is in the range of $w$ if $d_{f}+d_{o} \gg$ $d_{f}$ is not fulfilled, where $d_{o}$ is the object distance. So it is possible to design high quality imaging system in laboratory scale.

However, this technique has a limitation regarding the microwave power. Higher microwave power causes higher $\mathrm{X}$-ray emission from the plasma and in order to avoid the generation of stuck pixels, the lowest exposure time of the camera should be taken into account when the RF power is appointed.

More than 10 years ago (2002), the Atomki ECR group carried out and published for the first time space-resolved plasma diagnostics measurements by a pinhole X-ray camera. ${ }^{3,4}$ Recently (2014), new efforts were taken motivated by the unique features of this method to investigate the effect of different RF resonant modes to the plasma structure, the effect of the well-known fine frequency tuning. ${ }^{5}$ This paper aims to compare the two X-ray camera and detector setups, experimental techniques, and also the scientific goals of the experiments. 


\section{COMPARISON OF EXPERIMENTAL TECHNIQUES}

Both experiments (2002 and 2014) were carried out in the Atomki ECRIS Laboratory. Detailed description of the Atomki-ECRIS summarizing the special features of the ion source can be found in a recent paper. ${ }^{6} \mathrm{X}$-ray imaging was possible by the modification of the ion source. The guiding principle was to evolve setups which allow taking X-ray images and $\mathrm{m} / \mathrm{q}$ spectra simultaneously, providing information on exactly the same plasma. The X-ray camera was set on the axis of the injection side and was pumped by the vacuum system of the ion source. The area of the circle shape injection plate was divided into two regions in the ratio 3:2. The smaller area was used for microwave and gas injection, while the larger part was closed by stainless steel mesh to form closed resonant cavity and to provide transparency for imaging. Demagnification was necessary because the diameter of the plasma chamber is larger than the size of the CCD chip. Detailed comparison of the experimental parameters is shown by Table I.

Note here the main differences of the two campaigns. The goal of the 2002 experiment was to make the first ECR X-ray photos and to carry out simple studies on the effect of some setting parameters (magnetic field, extraction, disc voltage, gas mixing, etc.). In the present work, both the instrumentation and the scientific goals were much wider. We were focusing the question: how the plasma structure in the warm electrons domain is affected by different RF resonant modes? Silicon Drift Detector (SDD) and High Purity Germanium (HPGe) detectors were additionally installed (Fig. 1) to characterize

TABLE I. Comparison of the parameters of the experimental setups.

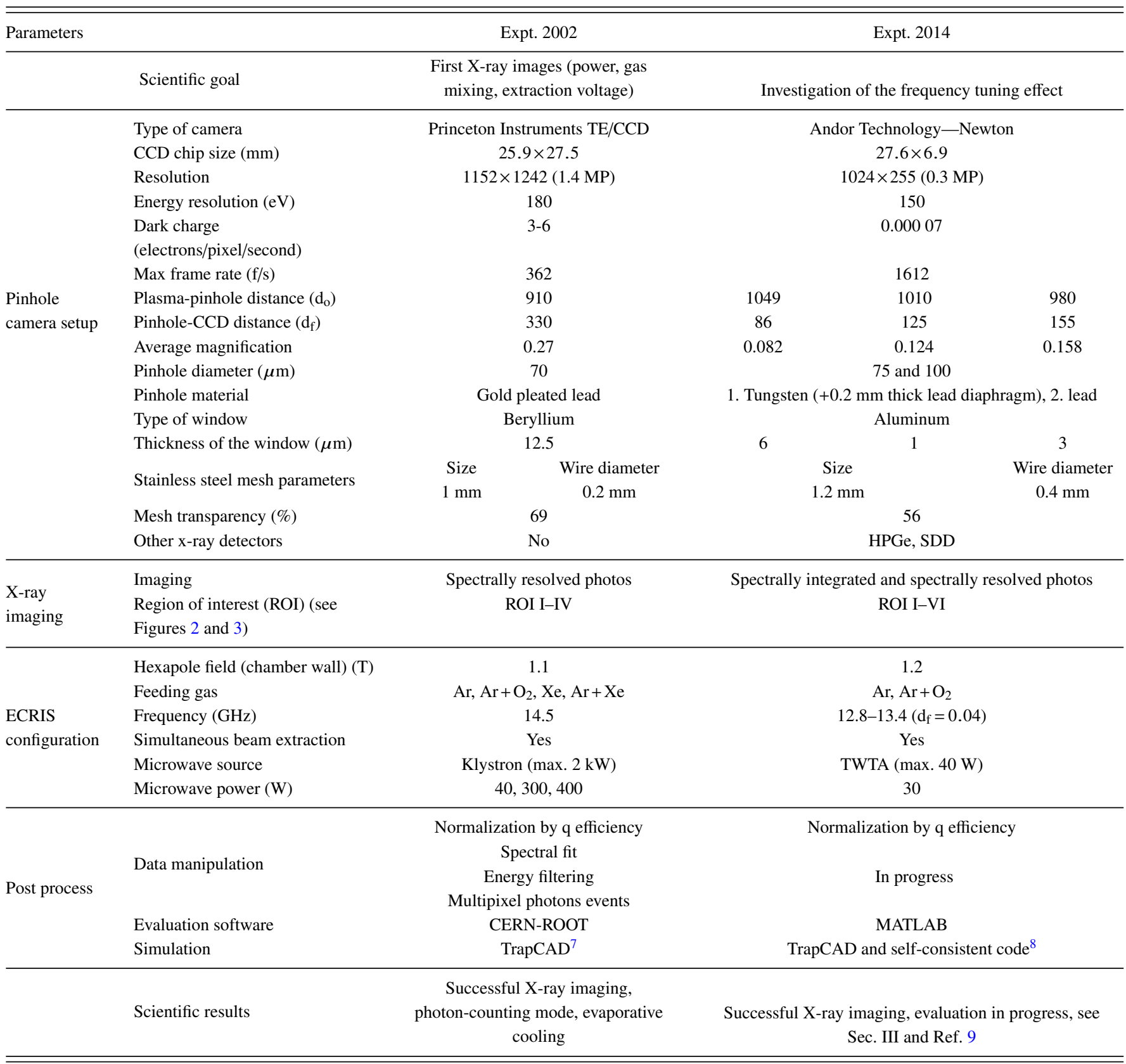




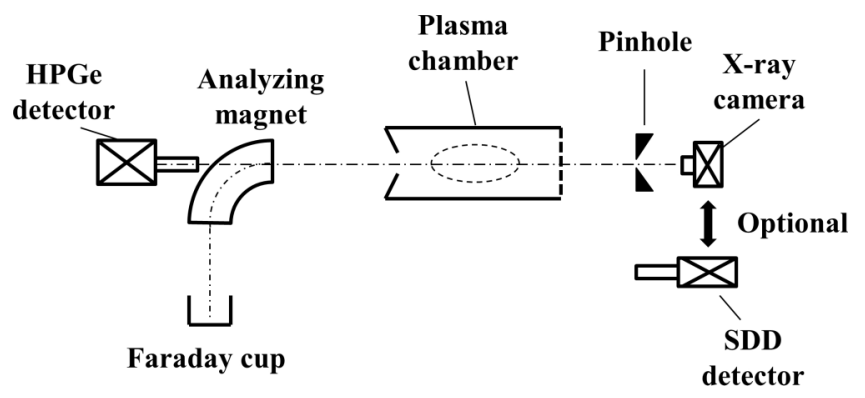

FIG. 1. Schematic drawing of the 2014 X-ray camera setup. Aluminum window was installed in front of the pinhole for filtering the low energy part of the EMW spectra.

the volumetric X-ray emission rate caused by the warm and hot electrons in the recent experiment. The scientific results obtained by using these two detectors are going to be published. ${ }^{9}$

There is a major conformity in the data acquisition techniques: photos were taken in photon counting mode in both cases. Thousands of images were taken with experimentally adjusted short (milliseconds) exposure time while each pixels registered either 0 or $1 \mathrm{X}$-ray events. Therefore, any individual pixel can be used as a single photon detector to spectrally resolve the plasma image. Because of the strong inhomogeneity of the plasma, region of interests (ROIs) were selected and the exposure time was settled by the intensity of the given region. The intensity of the other parts of the images was left out of consideration.

Typical X-ray plasma image exposed in 2002 with the selected ROIs can be seen in Fig. 2. Additionally, in 2014, spectrally integrated images were recorded with several seconds exposure time to investigate (only) the count rate corresponding to different parts of the plasma. Typical spectrally integrated X-ray plasma image can be seen in Fig. 3.

\section{RESULTS}

The results of the measurements with SDD and HPGe detectors in 2014 are published in an accompanying paper. ${ }^{9}$ Evaluation and detailed analysis of the data recorded with CCD pinhole camera are still in progress. The preliminary

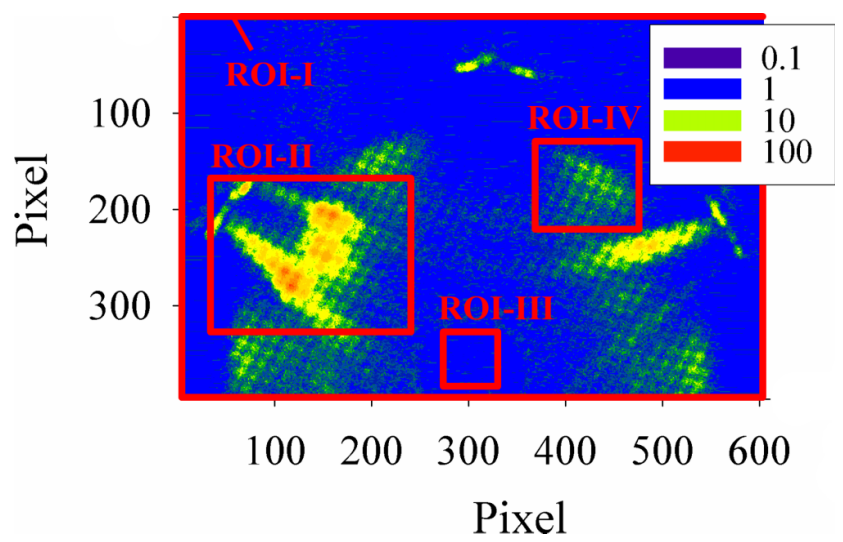

FIG. 2. X-ray image of argon plasma $\left(\mathrm{P}_{\mathrm{uw}}=40 \mathrm{~W}, \mathrm{f}=14.5 \mathrm{GHz}\right)$ recorded in 2002 with the typical ROIs. ROI-I: full size image, ROI-II: magnetic pole position, "star lobe," ROI-III: extraction hole, ROI-IV: plasma region.

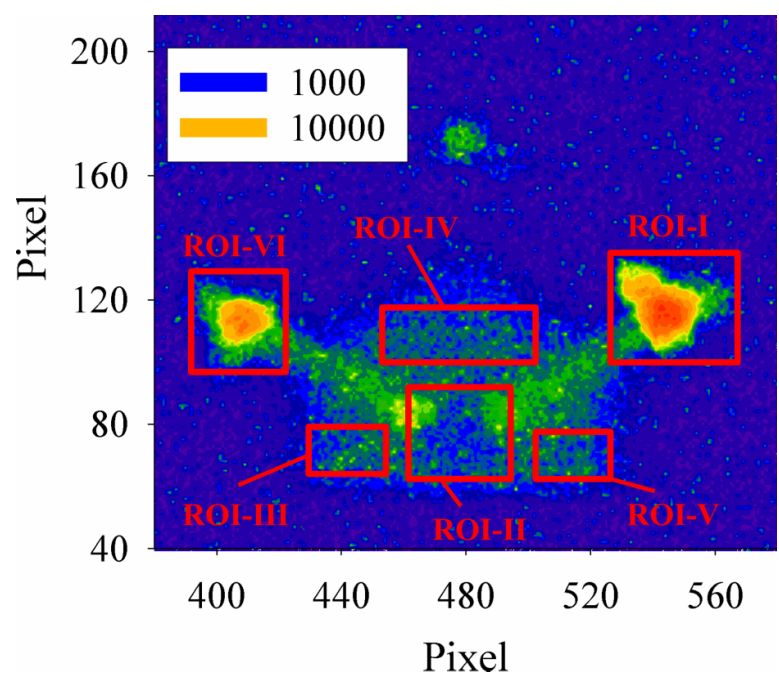

FIG. 3. Spectrally integrated X-ray image of argon plasma $\left(\mathrm{P}_{\mathrm{uw}}=30 \mathrm{~W}\right.$, $\mathrm{f}=12.84 \mathrm{GHz}$ ) recorded in 2014 with the typical ROIs. ROI-I and ROI-VI: magnetic pole, ROI-II: extraction hole, ROI-III-ROI-V: plasma region.

results coming from the pictures however are promising and shown here.

In order to explore the effect of the fine frequency tuning, spectrally integrated photos were taken of argon plasmas $\left(\mathrm{P}_{\text {inj }}=3.3 \times 10^{-6}\right.$ mbar $)$ as function of the injected RF frequency. Count rate in the ROI-I and ROI-VI was investigated corresponding to the electron losses on the superficies of the plasma chamber. Simultaneously, m/q spectra were recorded and the mean charge of the plasma $(\langle q\rangle)$ was assigned. As it is clearly visible in Fig. 4, the average charge (red circle) is fluctuating by the frequency and it is in strong correlation with the total count in ROI-I and ROI-VI (blue triangle).

In order to interpret the origin of the observed fluctuation, the spatial distribution of the lost (and non-lost) electrons in the ECRIS was calculated as function of the RF frequency by the TrapCAD simulation code. Details of the code are fully described elsewhere. $^{7}$

Formerly, the TrapCAD was successfully used for the interpretation of the fluctuation of the highly charged ions obtained in the HIMAC (Heavy Ion Medical Accelerator in Chiba) ECRIS in case of two close frequency heating by tuning

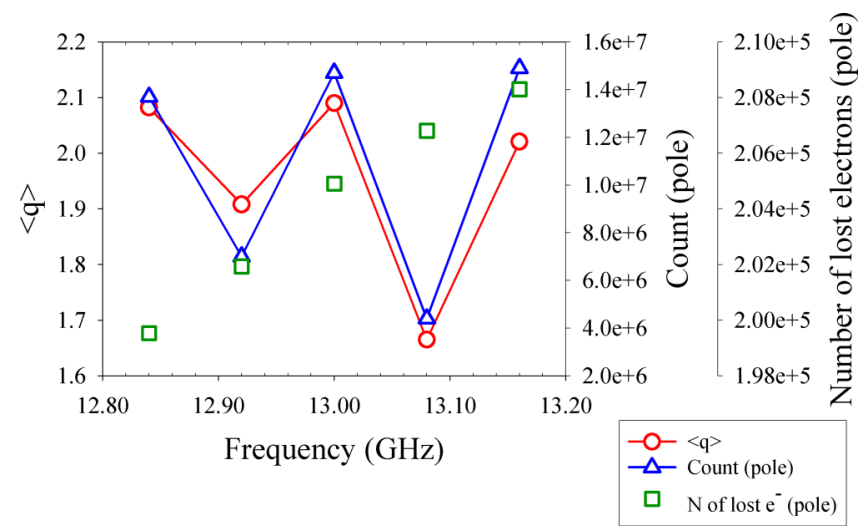

FIG. 4. Mean charge, sum of total count in ROI-I, ROI-VI, and the number of the lost electrons (based on TrapCAD) as function of the frequency. 
one of the injected rf frequency. ${ }^{10}$ The code is based on the one electron approach neglecting the particle-particle interactions. Circularly polarized plane wave is assumed to be propagating along the chamber axis.

$5 \times 10^{5}$ electrons were placed now with equal density into a thin layer developed by the closed resonance zone. The frequency of the microwave was set in the $12.84-13.16 \mathrm{GHz}$ range with steps of $80 \mathrm{MHz}$. The simulation time was $200 \mathrm{~ns}$; in real (CPU) time, the calculations lasted for $500 \mathrm{~h}$. At the end of the simulations, $\sim 40 \%$ of the electrons were still remained in the plasma and $\sim 60 \%$ were lost. The losses were divided into three main groups: electrons lost on the injection plate, lost in the extraction plate (plasma electrode), and lost on the tube-form plasma chamber wall.

The number of the electrons lost on the chamber wall (green square) as function of the RF frequency was plotted in Fig. 3 as well.

Result of the numerical simulation (the number of the electrons lost in poles) is not following the trend pointed by $\langle q\rangle$ and by ROI-I and ROI-VI. This discrepancy is very important by an interpretative point of view: it may be due to the modelled wave-plasma interaction in TrapCAD, which does not take into account eventual resonator effects and the impact of the cavity modes (and of modal density) on the plasma heating and confinement dynamics.

\section{ACKNOWLEDGMENTS}

The research leading to these results has received funding from the European Union Seventh Framework Program No. FP7/2007-2013 under Grant Agreement No. 262010-ENSAR. The EC is not liable for any use that can be made on the information contained herein. The author of this paper would like to thank all of their colleagues who took any part in the 2002 experiment (authors in Refs. 2 and 3).

${ }^{1}$ L. Kenéz, S. Biri, J. Karácsony, A. Valek, T. Nakagawa, K. E. Stiebing, and V. Mironov, Rev. Sci. Instrum. 73, 617 (2002).

${ }^{2}$ R. Rácz, S. Biri, and J. Pálinkás, Plasma Sources Sci. Technol. 20, 025002 (2011).

${ }^{3}$ S. Biri, A. Valek, T. Suta, E. Takács, Cs. Szabó, L. T. Hudson, B. Radics, J. Imrek, B. Juhász, and J. Pálinkás, Rev. Sci. Instrum. 75, 1420 (2004).

${ }^{4}$ E. Takács, B. Radics, C. I. Szabó, S. Biri, L. T. Hudson, J. Imrek, B. Juhász, T. Suta, A. Valek, and J. Pálinkás, Nucl. Instrum. Methods Phys. Res., Sect. B 235, 120 (2005).

${ }^{5}$ L. Celona, S. Gammino, G. Ciavola, F. Maimone, and D. Mascali, Rev. Sci. Instrum. 81, 02A333 (2010).

${ }^{6}$ S. Biri, R. Rácz, and J. Pálinkás, Rev. Sci. Instrum. 83, 02 A341 (2012).

${ }^{7}$ S. Biri, R. Rácz, and J. Pálinkás, IEEE Trans. Plasma Sci. 39, 2474 (2011).

${ }^{8}$ D. Mascali, G. Torrisi, L. Neri, G. Sorbello, G. Castro, L. Celona, and S. Gammino, Eur. Phys. J. D 69, 27 (2015).

${ }^{9}$ D. Mascali et al., "Electron Cyclotron Resonance Ion Source plasma characterization by X-ray spectroscopy and X-ray imaging," Rev. Sci. Instrum. (these proceedings).

${ }^{10}$ S. Biri, A. Kitagawa, M. Muramatsu, A. G. Drentje, R. Rácz, K. Yano, Y. Kato, N. Sasaki, and W. Takasugi, Rev. Sci. Instrum. 85, 02A931 (2014). 